\title{
PERFIL LINGÜÍSTICO EN NIÑOS Y ADOLESCENTES CON TRASTORNO POR DÉFICIT DE ATENCIÓN E HIPERACTIVIDAD (TDAH)
}

\author{
Esther Moraleda Sepúlveda \\ Facultad de Ciencias de la Salud \\ Universidad de Castilla-La Mancha \\ esther.moraleda@uclm.es \\ Lucía Fernández-Marcos \\ Facultad de Ciencias de la Salud \\ Universidad de Castilla-La Mancha \\ Jorge Sánchez Díaz \\ Facultad de Ciencias de la Salud \\ Universidad de Castilla-La Mancha \\ Patricia López Resa \\ Facultad de Ciencias de la Salud \\ Universidad de Castilla-La Mancha
}

Fecha de Recepción: 19 Julio 2018

Fecha de Admisión: 1 Octubre 2018

\section{RESUMEN}

Las habilidades lingüísticas aparecen como una dificultad específica en las personas con Trastorno por Déficit de Atención e Hiperactividad (TDAH). Los estudios realizados avalan que parecen existir problemas en las áreas de producción y comprensión del lenguaje. Por ello, el objetivo de este estudio fue conocer el perfil lingüístico que presentan los niños y adolescentes con TDAH. La muestra estuvo compuesta por 90 niños y adolescentes de entre 5 y 15 años divididos en dos grupos: 45 con TDAH y 45 con Desarrollo Típico, emparejados en edad cronológica. La evaluación del lenguaje se realizó a través de la prueba Celf-4. Los resultados muestran que las personas con TDAH muestran peores habilidades lingüísticas y, que, presentan dificultades más específicas en comprensión, así como en las subáreas relacionadas con la sintaxis. Como conclusión se destaca que las personas con TDAH necesitan recibir intervención lingüística como parte de su tratamiento integral.

Palabras clave: trastorno por déficit de atención e hiperactividad; niños; adolescentes; lenguaje 


\section{PERFIL LINGÜÍSTICO EN NIÑOS Y ADOLESCENTES CON TRASTORNO POR DÉFICIT DE ATENCIÓN E HIPERACTIVIDAD (TDAH)}

\section{ABSTRACT \\ LINGUISTIC PROFILE IN CHILDREN AND ADOLESCENTS WITH ATTENTION DEFICIT HYPE- RACTIVITY DISORDER}

Language skills appear as a specific difficulty in people with Attention Deficit Hyperactivity Disorder (ADHD). The studies carried out support that there seem to be problems in the areas of production and language comprehension. Therefore, the objective of this study was to know the linguistic profile presented by children and adolescents with ADHD. The sample consisted of 90 children and adolescents between 5 and 15 years old divided into two groups: 45 with ADHD and 45 with Typical Development, matched in chronological age. The evaluation of the language was carried out through the Celf-4 test. The results show that people with ADHD show worse language skills and that they have more specific difficulties in comprehension, as well as in the subareas related to syntax. In conclusion, it is emphasized that people with ADHD need to receive linguistic intervention as part of their integral treatment.

Keywords: attention deficit hyperactivity disorder; children; adolescent; language

\section{INTRODUCCIÓN}

El Trastorno por Déficit de Atención e Hiperactividad (en adelante TDAH) es un trastorno neurobiológico que se caracteriza por un patrón persistente de inatención y/o hiperactividad/ impulsividad que interfiere en la vida de la persona. La mayoría de estos síntomas están presentes antes de los 12 años y aparecen en todos los contextos (American Psychiatric Association, 2014). Si bien los datos de prevalencia varían en función de los estudios realizados, podemos encontrar una tasa de entre el 4 y el 10\% de casos en niños y adolescentes (Pascual-Castroviejo, 2008; Rodillo, 2015).

En cuanto a un perfil de lenguaje específico, los niños con TDAH, demuestran, en general, peores habilidades lingüísticas a todos los niveles en comparación con niños con Desarrollo Típico (DT en adelante) (Geurts, y Embrechts, 2008; Ygual, 2011). Además, estas dificultades en lenguaje y comunicación están presentes prácticamente desde las primeras etapas del desarrollo (Landa, 2007; Rapin, y Dunn, 2003) a través de un retraso en la adquisición del lenguaje (Hinshaw, 2002) y siguen produciéndose a lo largo de la adolescencia (Frazier, Youngstrom, Glutting, y Watkins, 2007; Semrud-Clikeman, Guy, Griffin, y Hynd, 2000), lo que da lugar a problemas sociales posteriormente (Snowling, Bishop, Stothard, Chipchase, y Kaplan, 2006). Algunos autores atribuyen directamente una relación direccional a la presencia de TDAH y la aparición de un trastorno del lenguaje (Bruce, Thernlund, y Nettelbladt, 2006; Cohen, Vallance, Barwick, Menna, Horodezky, y Isaacson, 2000; Geurts, y Embrechts, 2008; Helland, Biringer, Helland, y Heimann, 2012; Helland, Posserud, Helland, Heimann, y Lundervold, 2016). Cada vez son más las investigaciones que encuentran características similares a nivel lingüístico entre el TDAH y el Trastorno "Específico" del Lenguaje (Bellani, Moretti, Perlini y Brambilla, 2011; Hutchinson, Bavin, Efron y Sciberras, 2012; Redmond, 2004)

Las personas con TDAH tienen problemas en contenido, forma y uso del lenguaje (Rapin, y Dunn, 2003). Muestran grandes dificultades en áreas como la conciencia fonológica, articulación de palabras, fluidez de habla, morfología y sintaxis (Geurts, y Embrechts, 2008, Kim y Kaiser, 2000; Vaquerizo, Estévez y Pozo, 2005). Por ejemplo, presentan una producción fonológica más pobre (Cohen et al, 2000). En relación a la morfosintaxis, el rendimiento es menor en tareas de expresión morfológica donde se desarrollan habilidades relacionadas con la utilización de morfemas de número, utilización del tiempo pasado tanto en verbos regulares como irregulares y utilización del comparativo y superlativo en comparación con niños con Desarrollo Típico, así como en sintaxis (Brook, y Boaz, 2005; Mahone, Koth, Schuerholz, Singer, y Denckla, 1999; Mathers, 2006; Purvis, y Tannock, 1997). Además, las personas con TDAH presentan una menor capacidad de procesamiento semántico (Tannock, Martinussen, y Frijters, 2000). Pero, especialmente, se han mostrado difi- 
cultades en habilidades narrativas, ya que producen narraciones más cortas, no son capaces de organizar bien la información y les cuesta dar cohesión a sus enunciados (Camarta y Gibson, 1999; Etchepareborda, 2003; Geurts, Verte, Oosterlaan, Roeyers y Sergeant, 2004; Rumpf, Kamp-Becker, Becker y Kauschke, 2012). Una de las causas parece radicar entre otras cuestiones, en las grandes dificultades a la hora de memorizar estímulos auditivamente (Crespo y Narbona, 2003).

Asimismo, parece que los problemas en memoria de trabajo dan lugar a que no sólo esté afectado el componente productivo del lenguaje, sino que la comprensión también se encuentra alterada (Bignell y Cain, 2007; Carrow- Woolfolk, 2014; Jonsdottir, Bouma, Sergeant y Scherder, 2005; Mclnnes, Humphries, Hogg-Johnson y Tannock, 2003). Además, las dificultades específicas en comprensión oral dan paso después a dificultades de comprensión escrita (Cutting y Scarborough, 2006; Miller, Keenan, Betjemann, Willcutt, Pennington \& Olson, 2013; Sesma, Mahone, Levine, Eason \& Cutting, 2009;).

\section{OBJETIVO}

El objetivo de este trabajo es ahondar acerca de las características lingüísticas de este colectivo, teniendo en cuenta, que la mayor parte de las investigaciones realizadas hasta el momento son de habla inglesa y aún son escasos los estudios dedicados a conocer el perfil lingüístico en niños y adolescentes con TDAH de lenguaje castellana.

\section{MÉTODO}

\section{Participantes}

En este estudio participaron 90 niños y adolescentes de entre 5 y 15 años correspondientes a dos grupos de edad. El primero, son 45 personas con TDAH, todos con diagnóstico confirmado por un especialista médico (13 mujeres y 32 varones) con una media de edad de 9.59 años (Desviación Típica=3.35). El segundo grupo estaba formado por personas con Desarrollo Típico (25 mujeres y 20 varones) emparejados en edad cronológica ( $X=9.24$; Desviación Típica= 3.29).

\section{Instrumentos}

Para llevar a cabo la evaluación lingüística se utilizó la versión completa de la prueba estandarizada CELF-4, Spanish Clinical Evaluation of Language Fundamentals-4 (Wiig, Secord y Semel, 2006). Esta prueba se administra a poblaciones comprendidas entre 5 y 22 años de edad y evalúa el nivel de lenguaje en varias áreas: morfología, sintaxis, semántica y pragmática, dando lugar finalmente a puntuaciones que se recogen en las siguientes áreas: base del lenguaje, lenguaje receptivo, lenguaje expresivo, contenido del lenguaje, estructura del lenguaje y memoria de trabajo. Además, el test está compuesto a su vez de varias subpruebas (conceptos y siguiendo direcciones, formulación de oraciones, recordando oraciones, clases de palabras receptivo, clases de palabras expresivo, vocabulario expresivo, recuerdo de números total y secuencias familiares) que permiten obtener una edad equivalente correspondiente.

\section{Procedimiento}

En primer lugar, se contactó con diversas asociaciones de TDAH y clínicas privadas con el fin de conocer si estarían interesados en la participación en este estudio. Una vez los centros accedieron y se confirmaron los sujetos de la muestra, se les envió el documento de consentimiento informado. Al mismo tiempo, se contactó con diferentes centros educativos para conseguir la muestra de personas con DT. Tras la firma de dicho documento en ambos casos, se llevó a cabo la evaluación del lenguaje individualizada mediante la prueba CELF-4. Todos los participantes fueron evaluados de manera individual en una sesión. 


\section{PERFIL LINGÜÍSTICO EN NIÑOS Y ADOLESCENTES CON TRASTORNO POR DÉFICIT DE ATENCIÓN E HIPERACTIVIDAD (TDAH)}

\section{RESULTADOS}

En la Tabla I, en la que se muestran las puntuaciones obtenidas en relación a los dos grupos de participantes. Se puede observar cómo el grupo de niños y adolescentes con TDAH puntúan por debajo de los niños con DT en las diferentes áreas evaluadas. Al ser datos paramétricos se utilizó la prueba t de Student.

Asimismo, las diferencias son significativas en todas las áreas: base del lenguaje (t $(44)=-5.99$, $p<.01$ ), lenguaje receptivo ( $t(44)=-6.49, p<.01$ ), lenguaje expresivo ( $t(44)=-6.79, p<.01)$, contenido del lenguaje $(\mathrm{t}(44)=-6.21, \mathrm{p}<.01)$, estructura del lenguaje $(\mathrm{t}(44)=-5.98, \mathrm{p}<.01)$ y memoria de trabajo $(\mathrm{t}(44)=-4.77, p<.01$ ). Teniendo en cuenta que el test considera que los resultados corresponderían a la norma a partir de 100 puntos, los niños y adolescentes con TDAH puntúan por debajo de la norma en base del lenguaje, lenguaje receptivo, lenguaje expresivo y estructura del lenguaje.

Posteriormente, se analizaron los datos en función de las subáreas que componen el test. Los resultados hallados muestran que las diferencias son significativas en las subáreas de recordando oraciones (t $(44)=-5.17, p<.01$ ) y entendiendo oraciones (t $(44)=-3.38, p<.01$ ). En el caso de la subárea clases de palabras receptivo, se utilizó la prueba $U$ de Mann-Whitney dado que los datos eran no paramétricos. En esta subárea también se encontraron diferencias significativas entre ambos grupos $(p<.01)$.

Tabla I. Puntuaciones medias en las áreas lingüísticas

\begin{tabular}{lcr}
\hline & Personas con TDAH & Personas con DT \\
\hline Base del lenguaje & $93.80(21.9)$ & $118.96(17.5)$ \\
Lenguaje receptivo & $88.11(15.6)$ & $109.36(15.3)$ \\
Lenguaje expresivo & $98.40(13.1)$ & $120.22(16.9)$ \\
Contenido del lenguaje & $100.73(16.8)$ & $119.89(11.9)$ \\
Estructura del lenguaje & $94.71(11.7)$ & $113.93(18.1)$ \\
Memoria de trabajo & $101.11(17.4)$ & $118.36(16.8)$ \\
\hline
\end{tabular}

Desviaciones típicas entre paréntesis

\section{DISCUSIÓN Y CONCLUSIONES}

Los resultados encontrados revelan que en general, Ios niños y adolescentes con TDAH muestran peores habilidades lingüísticas en comparación con los niños con DT, confirmando lo encontrado en estudios anteriores (Andreou, Agapitou \& Karapetsas, 2005; Geurts y Embrets, 2008; Rumpf et al., 2012). Además, y teniendo en cuenta la prueba administrada (Celf-4) parecen mostrar las mayores dificultades en el área de lenguaje receptivo. Sin embargo, no ocurre así en el área de memoria de trabajo, donde las personas con TDAH evaluadas se sitúan en un nivel medio, en contraposición a otras investigaciones realizadas (Dahlin, 2011; Klingberg, Forssberg \& Westerberg, 2002; Martinussen, Hayden, Hogg-Johnson \& Tannock, 2005). Esto nos lleva a dos posibles interpretaciones. Por un lado, en las personas con TDAH podría existir una disociación entre la memoria de trabajo y la comprensión oral que habría que analizar más específicamente en futuras investigaciones. Por otro lado, sería interesante ahondar en las habilidades de comprensión oral en este colectivo, ya que parece ser un área de especial dificultad como apuntan otros trabajos revisados (Bignell y Cain, 2007; Bruce et al., 2006; McInnes et al., 2003; Wassenberg, Hendriksen, Hurks, Feron, Vles, \& Jolles, 2010) que, además, debería ser tenida en cuenta como prioritaria de cara a planificar el tratamiento y la intervención posterior. Asimismo, es importante destacar que, además del lenguaje receptivo, hay tres subáreas más de lenguaje expresivo que sitúan los resultados de las personas con TDAH por debajo de la norma: base del lenguaje, lenguaje expresivo y estructura del 
lenguaje. Estas tres subáreas están relacionadas principalmente con el área sintáctica e indicarían una especial dificultad a la hora de organizar el lenguaje, tal y como apuntan otros estudios (Redmond, 2004; Delgado, Etchepareborda, Baker y Rubiales, 2013; Gallardo-Paúls, Moreno, Roca, \& Pérez, 2012; Raggi y Chronis, 2006; Väisänen, Loukusa, Moilanen, \& Yliherva, 2014).

No obstante, a la hora de analizar específicamente las subáreas del test, es importante destacar cómo las principales dificultades de las personas con TDAH se han encontrado en las pruebas que no conllevan la utilización de lenguaje oral. Es decir, los mayores déficits se encuentran en las subáreas implícitas en el lenguaje comprensivo (especialmente en entendiendo párrafos y clases de palabras receptivo). Estos datos nos llevan una vez más, a la confirmación de que el procesamiento y la comprensión de información auditiva por parte de este colectivo se produce de manera deficitaria (Beitchman, Hood, Rochon, Peterson, Mantini, y Majumdar, 1989; Haghshenas, Hosseini, y Aminjan, 2014; Quinn, 2003; Tinius 2003).

En conclusión, las personas con TDAH son susceptibles de intervención lingüística a lo largo de todo su desarrollo, dado que cada vez son más las investigaciones y los casos que demuestran que presentan dificultades lingüísticas específicas que habría que seguir analizando, no sólo a nivel oral, sino también en la vertiente escrita.

\section{BIBLIOGRAFÍA}

Andreou, G., Agapitou, P. \& Karapetsas, A. (2005). Verbal skills in children with ADHD. European Journal of Special Needs Education, 20(2), 231-238.

American Psychiatric Association. (2014). Guía de consulta de los criterios diagnósticos del DSM5: Spanish Edition of the Desk Reference to the Diagnostic Criteria From DSM-5. Washington, DC: American Psychiatric Pub.

Beitchman, J.H., Hood, J., Rochon, J., Peterson, M., Mantini, T. \& Majumdar, S. (1989). Empirical classification of speech/language impairment in children. I. Identification of speech/language categories. Journal of the American Academy of Child and Adolescent Psychiatry, 28 (1), 112117

Bellani, M., Moretti, A., Perlini, C. \& Brambilla, P. (2011). Language disturbances in ADHD. Epidemiology and Psychiatric Sciences, 20, 311-315.

Bignell, S. \& Cain, K. (2007). Pragmatic aspects of communication and language comprehension in groups of children differentiated by teacher ratings of inattention and hyperactivity. British Journal of Developmental Psychology, 25(4), 499-512.

Brook, U., \& Boaz, M. (2005). Attention deficit and hyperactivity disorder (ADHD) and learning disabilities (LD): adolescents perspective. Patient Education and Counseling, 58(2), 187-191.

Bruce, B., Thernlund G., \& Nettelbladt, U. (2006). ADHD and language impairment. A Study of the parent questionnaire FTF (Five to Fifteen). European Child y Adolescent Psychiatry, 15(1), 5360.

Camarta, S. M., \& Gibson, T. (1999). Pragmatic language deficits in attention-deficit hyperactivity disorder (ADHD). Mental Retardation Developmental Disabilities research reviews, 5(3), 2072014.

Carrow-Woolfolk, E. (2014). TACL-4: Test for Auditory Comprehension of Language-Fourth Edition. Pro Editions.

Cohen, N., Vallance, D., Barwick, M., Menna, R., Horodezky, N. \& Isaacson, L. (2000). The Interface between ADHD and Language Impairment: An Examination of Language, Achievement and Cognitive Processing. Journal of Child Psychology and Psychiatry, 41, 353-362.

Crespo-Eguílaz, N. \& Narbona, J. (2003). Perfiles clínicos evolutivos y transiciones en el espectro 
del trastorno específico del desarrollo del lenguaje. Revista de Neurología. 36(1), 29-35.

Cutting, L. \& Scarborough, H. (2006). Prediction of Reading Comprehension: Relative Contributions of Word Recognition, Language Proficiency, and Other Cognitive Skills Can Depend on How Comprehension Is Measured. Scientific Studies of Reading, 10(3), 277-299.

Dahlin, K. (2011). Effects of working memory training on reading in children with special needs. Reading and Writing, 24(4), 479-491.

Delgado-Mejía, I. D., Etchepareborda, M. C., Bakker, L. \& Rubiales, J. (2013). Análisis de los sistemas de procesamiento secuencial y simultáneo en niños con diagnóstico de trastorno por déficit de atención con hiperactividad. Anuario de Proyectos e Informes de Becarios de Investigación, 10 (10), 842-849.

Etchepareborda, M.C. (2003). La intervención en los trastornos disléxicos:entrenamiento de la conciencia fonológica. Revista de neurología, 36 (1), 13-19.

Frazier, T., Youngstrom, E., Glutting, J. \& Watkins, M. (2007). ADHD and achievement: Meta-analysis of the child, adolescent, and adult literatures and a concomitant study with college students. Journal of Learning Disabilities, 40, 49-65.

Gallardo-Paúls, B., Moreno, V., Roca, P. \& Pérez, J.L., (2012). Complejidad sintáctica y textual en niños con trastorno por déficit de atención/hiperactividad. Revista de Neurologia, 51(4), 131135.

Geurts, H. \& Embrechts, M. (2008). Language profiles in asd, sli and ADHD. Journal of autism and Developmental Disorders, 38, 1931-1943.

Geurts, H.M., Verte, S., Oosterlaan, J., Roeyers, H. \& Sergeant, J.A. (2004) How specific are executive functioning deficits in attention deficit hyperactivity disorder and autism? Journal of Child Psychology and Psychiatry, 45(4), 836-854.

Haghshenas, S., Hosseini, M. S., \& Aminjan, A. S. (2014). A possible correlation between vestibular stimulation and auditory comprehension in children with attention-deficit/hyperactivity disorder. Psychology Neuroscience, 7 (2), 159-162.

Helland, W. A., Biringer, E., Helland, T. \& Heimann, M. (2012). Exploring language profiles for children with ADHD and children with Asperger syndrome. Journal of Attention Disorders, 16(1), 34-43.

Helland, W.A., Posserud, M.B., Helland, T., Heimann, M. \& Lundervold, A.J. (2016). Language Impairments in Children With ADHD and in Children With Reading Disorder. Journal of Attention Disorders, 20(7), 581-589

Hinshaw, S. (2002). Preadolescent girls with attentiondeficit/hyperactivity Disorder: I Background characteristics, comorbidity, cognitive and social functioning, and parenting practices. Journal of Consulting and Clinical Psychology, 70, 1086-1098.

Hutchinson, E., Bavin, E., Efron, D. \& Sciberras, E., (2012). A comparison of working memory profiles in school-aged children with Specific Language Impairment, Attention Deficit/Hyperactivity Disorder, Comorbid SLI and ADHD and their typically developing peers. Child Neuropsychology, 18(2), 190-207.

Jonsdottir, S., Bouma, A., Sergeant, J. \& Scherder, E. (2005). The impact of specific language impairment on working memory in children with ADHD combined subtype. Archives of Clinical Neuropsuchology 20(4), 443-456.

Kim, H. \& Kaiser, A., (2000). Language Characteristics of Children with ADHD. Journals Sage, 21 (3), 154-165.

Klingberg, T., Forssberg, H. \& Westerberg, H. (2002). Training of Working Memory in Children With ADHD. Journal of Clinical and Experimental Neuropsychology 24(6), 781-791. 
Landa, R. (2007). Early communication development and intervention for children with autism. Mental Retardation and Developmental Disabilities Research Reviews 13 (1), 16-25.

Mahone, E. M., Koth, C., Schuerholz, L., Singer, H. S., \& Denckla, M. B. (1999). Clustering and disinhibition on fluency and recall measures in Tourette syndrome and/or ADHD [Abstract]. Journal of the International Neuropsychological Society, 5, 98.

Martinussen, R., Hayden, J., Hogg-Johnson, S., \& Tannock, R. (2005). A Meta-Analysis of Working Memory Impairments in Children with Attention-Deficit/Hyperactivity Disorder. Journal of the American Academy of Child and Adolescent Psychiatry, 44, 377-384.

Mathers, M. E. (2006). Aspects of language in children with ADHD: Applying functional analyses to explore language use. Journal of Attention Disorders, 9(3), 523-533.

Mclnnes, A., Humphries, T., Hogg-Johnson, S., \& Tannock, R. (2003). Listening comprehension and working memory are impaired in attention-deficit hyperactivity disorder irrespective of language impairment. Journal of abnormal child psychology, 31(4), 427-443.

Miller, A., Keenan, J., Betjemann, R., Willcutt, E., Pennington, B., Olson, R. (2013). Reading comprehension in children with ADHD: cognitive underpinnings of the centrality déficit. Journal of Abnormal Child Psychology. 41 (3), 473-483.

Pascual-Castroviejo, I. (2008). Trastornos por déficit de atención e hiperactividad (TDAH). Neuropediatria: 140-150. Journal of Abnormal Child Psychology, 31(4), 427-443.

Purvis, K. L., \& Tannock, R. (1997). Language abilities in children with attention deficit hyperactivity disorder, reading disabilities, and normal controls. Journal of abnormal child psychology, 25(2), 133-144.

Quinn, C.A. (2003). Detection of malingering in assessment of adult ADHD. Archives of Clinical Neuropsychology, 18 (4), 379-395.

Rapin, I. \& Dunn, M. (2003). Update on the language disorders of individuals on the autistic spectrum. Brain and Development, 25 (3), 166-172.

Redmond, S.M. (2004). Conversational profiles of children with ADHD, SLI and typical development. Clinical Linguistics \& Phonetics, 18, 107-125.

Rodillo, E. (2015). Trastorno por déficit de atención e hiperactividad (TDAH) en adolescentes. Revista Médico Clínica las Condes, 26 (1), 52-59.

Rumpf, AL., Kamp-Becher, I. Becker, K. \& Kauschke, C. (2012) Narrative competence and internal state language of children with Asperger Syndrome and ADHD. Research in Developmental Disabilities, 33(5), 1395-1407.

Semrud-Clikeman, M., Guy, K., Griffin, J.D., \& Hynd, G.W. (2000). Rapid naming deficits in children and adolescents with reading disabilities and attention deficit hyperactivity disorder. Brain and Language, 74(1), 70-83.

Sesma, H., Mahone, E., Levine, T., Eason, S. \& Cutting, L. (2009). The contribution of executive skills to reading comprehension. Child Neuropsychology, 15(3), 232-246.

Snowling, M. J., Bishop, D. V. M., Stothard, S. E., Chipchase, B., \& Kaplan, C. (2006). Psychosocial outcomes at 15 years of children with a preschool history of speech-language impairment. Journal of Child Psychology and Psychiatry, 47 (8), 759-765.

Tannock, R., Martinussen, R. \& Frijters, J. (2000). Naming Speed Performance and Stimulant Effects Indicate Effortful, Semantic Processing Deficits in Attention Deficit/Hyperactivity Disorder. Journal of Abnormal Child Psycholohy, 28 (3), 237-252.

Tinius, T.P. (2003). The intermediate visual and auditory continuous performance test as a neuropsychological measure. Archives of Clinical Neuropsychology, 18 (2), 199-214.

Väisänen, R., Loukusa, S., Moilanen, I., \& Yliherva, A. (2014). Language and pragmatic profile in 
children with ADHD measured by Children's Communication Checklist 2nd edition. Logopedics Phoniatrics Vocology, 39(4), 179-187.

Vaquerizo-Madrid, J., Estévez-Díaz, F. \& Pozo-García, A., (2005). El lenguaje en el trastorno por déficit de atención con hiperactividad: competencias narrativas. Revista Neurológica, 41 (1), 83-89.

Ygual, A. (2011). Los trastornos del lenguaje en el trastorno por déficit de atención con hiperactividad (TDAH). Revista de Logopedia, Foniatría y Audiología, 31 (4), 181-182.

Wassenberg, R., Hendriksen, J., Hurks, P. Feron, F., Vles, J. \& Jolles, J. (2010). Speed of language comprehension is impaired in ADHD. Journal of Attention Disorders, 13 (4), 374-385.

Wiig, E., Secord, W. A, \& Semel, E., (2006) Clinical evaluation of language fundamentals - Preschool second. UK edition. London: Harcourt Assessment. 